\title{
Efeitos do uso de um programa cinesioterapêutico na força de preensão manual de idosas
}

\author{
Effects of using a program kinesiotherapeutic in grip strength manual of elderly
}

\author{
Thaisa Veloso Mendonça ${ }^{1}$, Adriana Sousa Rego ${ }^{1}$, Flor da Maria Araújo Mendonça \\ Silva ${ }^{2}$, José Newton Lacet Vieira ${ }^{3}$
}

Resumo: Objetivo: Verificar os efeitos do uso de um programa cinesioterapêutico na força de preensão manual de idosas. Materiais e Métodos: Estudo experimental, analítico realizado no período de outubro/novembro de 2013, no clube de repouso Irmã Dulce e na Associação Comunitária da Vila Cruzado, tendo envolvido 30 idosas pertencentes a 02 grupos de convivências, com idade igual ou superior a 60 anos, sendo que 20 idosas fizeram parte de um grupo experimental e 10 idosas fizeram parte de um grupo controle. Todas foram avaliadas quanto a força muscular máxima de preensão manual, com uso do equipamento dinamômetro mecânico. Em seguida, apenas o grupo de 20 idosas iniciou o programa cinesioterapêutico. Após 02 meses, todas as idosas foram reavaliadas. Para análise dos dados foi usado o programa estatístico SPSS 18.0. $\mathrm{Na}$ estatística descritiva, as variáveis quantitativas com distribuição simétrica são apresentadas em média e desvio padrão e as assimétricas em mediana, mínimo e máximo. Para a análise do efeito do programa cinesioterapêutico na força de preensão manual de idosas e na comparação intergrupos foi utilizado o teste de Wilcoxon. $\mathrm{Na}$ análise da relação entre força de preensão manual e faixa etária das idosas submetidas ao programa cinesioterapêutico foi utilizado o Kruskal-Wallis, sendo considerado significativo $p<0,05$. Resultados: Não foi verificada diferença significativa com relação à idade e peso corporal, por parte dos 02 grupos de idosas avaliadas. Foi verificada diferença estatisticamente significativa apenas no grupo de idosas submetidas ao programa cinesioterapêutico, quanto à força de preensão manual. Conclusão: Idosas submetidas a um programa cinesioterapêutico, mesmo que durante um curto período, apresentam aumento da força de preensão manual.

Palavras-chave: Programa. Força muscular. Idosas.

Abstract: Objective: To investigate the effects of using a kinesiotherapeutic program in handgrip strength of older. Materials and Methods: Experimental, analytical conducted between October-November 2013 in the rest of Sister Dulce Club and Community Association Village Cross, and involved 30 women belonging to 02 groups of cohabitation, aged 60 or over years, and 20 elderly women were part of an experimental group and 10 elderly were part of a control group. All were evaluated for maximum muscle grip strength, using the dynamometer equipment mechanic. Then, only the group of 20 elderly kinesiotherapeutic started the program. After 02 months, all participants were reassessed. For data analysis we used the SPSS 18.0 statistical program. Descriptive statistics, quantitative variables with symmetric distribution are presented as mean and standard deviation and asymmetric median, minimum and maximum. To analyze the effect of kinesiotherapeutic in handgrip strength of older and intergroup comparison program, the Wilcoxon test was used. In analyzing the relationship between force and manual age of older kinesiotherapeutic program submitted to hold the Kruskal-Wallis test was used, being considered significant $p<0.05$. Results: There was significant difference in age and body weight, by the 02 groups of elderly women. Statistically significant difference was observed in the group of elderly submitted to kinesiotherapeutic program, as the handgrip. Conclusion: Elderly undergone kinesiotherapeutic program, even for a short period, showed increased grip strength.

Keywords: Program. Muscle strength. Elderly.

\footnotetext{
${ }_{1}^{1}$ Departamento de Fisioterapia. Universidade Ceuma

2 Departamento de Psicologia. Universidade Ceuma

${ }^{3}$ Departamento de Fisioterapia. Universidade Ceuma. Endereço para correspondência:

Avenida Nova lorque, Q-5 E, C-03, Central Parque, Araçagy, São José de Ribamar-MA. CEP:

65.110-000. Fone: (98) 981109633.
} 


\section{Introdução}

O envelhecimento pode ser compreendido como um fenômeno biológico, que envolve a perda da capacidade funcional necessária para a realização das atividades de sua vida diária. Entretanto, devem ser levadas em conta as mudanças naturais desse processo. Assim, o envelhecimento bem-sucedido está associado a uma importante mudança ideológica que não considera a velhice como sinônimo de perda, doença, inatividade e algo contrário ao desenvolvimento. ${ }^{1}$

Em decorrência desse processo, ressalta-se a necessidade de suprir as carências que a população idosa possa apresentar, em especial no que tange ao acesso aos serviços de saúde. Diante da realidade inquestionável das transformações demográficas iniciadas no último século, em que se observa uma população cada vez mais envelhecida, evidencia-se a importância de garantir aos idosos não só uma sobrevida maior, mas também uma boa qualidade de vida. $^{2}$

A aquisição e a manutenção de níveis moderados de força muscular são importantes para a realização das atividades da vida diária (AVDs) das idosas, tais como, carregar pesos, subir escadas, levantar-se da cadeira, visto que o treinamento de força é capaz de minimizar os efeitos deletérios do envelhecimento, tornando-as independentes para a realização de tarefas diárias. $^{3}$

O processo de envelhecimento biológico determina alterações no aparelho locomotor, que causam limitações às atividades da vida diária e, assim, comprometem a qualidade de vida da pessoa que envelhece. A diminuição do nível de atividade pode levar o idoso a um estado de fragilidade e de dependência. Evidências atuais demonstram que a atividade física traz benefícios à saúde do idoso, mantendo independência funcional. ${ }^{4}$

A redução da massa e força muscular decorrentes do envelhecimento, ou sarcopenia, ocorre mesmo no idoso saudável e é considerada como o fator que contribui de forma mais significativa para a perda de independência e funcionalidade nessa faixa etária. ${ }^{5}$

A Fisioterapia gerontológica tem merecido papel de destaque no atendimento ao paciente idoso, atuando em nível de promoção, prevenção, tratamento, reabilitação e adaptação da funcionalidade do idoso. Ela adéqua suas técnicas às características próprias do organismo em questão, contribuindo para a autonomia e qualidade de vida desta população. ${ }^{6}$

Em função dessas alterações aumenta a probabilidade de quedas associada à diminuição da capacidade funcional. Estudos têm sido realizados buscando-se os benefícios da atividade física nos idosos, além da implantação de programas de exercícios, que demonstram que além de diminuir o número de quedas, também melhoram o equilíbrio, a força muscular e a marcha, além da melhora psicológica. ${ }^{7}$

As pesquisas que avaliam os níveis de atividade física, autonomia funcional e qualidade de vida na população de idosos no Brasil estão crescendo cada vez mais diante do visível envelhecimento da 
população, porém pesquisas dessa magnitude ainda são escassas em alguns municípios. ${ }^{8}$

A consciência dos efeitos que a idade exerce sobre a capacidade de aprender e executar as habilidades motoras coloca 0 fisioterapeuta em condições de ajudar o geronte, no sentido de aumentar sua participação numa série de atividades de lazer ou profissionais, além de orientá-lo a desenvolver sua capacidade de adaptação, em resposta às limitações físicas. ${ }^{9}$

A força de preensão manual é capaz de medir a força muscular máxima utilizada por um membro superior dominante, por meio de um dinamômetro. $\mathrm{O}$ instrumento fornece uma leitura rápida e direta da força isométrica e é adaptável a diferentes tamanhos de mão. ${ }^{10}$

Considerando-se que a fisioterapia é uma ciência que atua no atendimento a pessoas idosas; tornou-se este estudo relevante, pois a avaliação da força de preensão manual é uma importante ferramenta para identificar a necessidade da realização de programas de cinesioterapia que poderão ser utilizados para minimizar a perda, ou aumentar a força muscular. Sendo assim o objetivo deste estudo foi verificar os efeitos do uso de um programa cinesioterapêutico na força de preensão manual de idosas.

\section{Materiais e métodos}

Tratou-se de um estudo experimental, analítico. ${ }^{11} \mathrm{~A}$ população foi composta por 30 idosas, por conveniência, com idade igual ou superior a 60 anos, do sexo feminino, divididas em 2 grupos de estudo, sendo 20 idosas de um grupo de convivência pertencentes ao Clube de Repouso Irmã Dulce, que fizeram parte do grupo experimental (GE), e outras 10 idosas de um grupo de convivência pertencentes à Associação Comunitária da Vila Cruzado, que fizeram parte do grupo controle (GC), no período de outubro a novembro de 2013.

Para coleta de dados, foi feita inicialmente uma reunião com os grupos de idosas de convivência, que pertencem ao clube de Repouso Irmã Dulce, e a ACOVIC.

Em seguida foi feita a seleção das idosas por critérios de inclusão referente a participação apenas mulheres com idade igual ou superior a 60 anos pertencentes a um grupo de convivência; e de exclusão referente às idosas que apresentassem ferimentos graves em fase de recuperação, ou sequelas de fratura que impossibilitasse a realização do teste de força muscular, e consequentemente a execução do programa cinesioterapêutico.

Posteriormente foi dado início a avaliação da força muscular, com aparelho dinamômetro mecânico ${ }^{10}$ Gold Standard, modelo POWER DIN PRO, fabricado pela empresa CEFISE - Biotecnologia Esportiva, com Software N2000 Pro, para avaliação da força muscular máxima de preensão manual das 30 pessoas idosas, sendo que as 20 idosas do grupo experimental (GE) foram submetidas a avaliação da força muscular máxima de preensão manual, antes e após a aplicação de um programa cinesioterapêutico, com intervalo de dois meses, enquanto que as 10 pessoas idosas 
do grupo controle (GC), fizeram apenas a avaliação da força muscular máxima de preensão manual, durante o mesmo período.

Todas idosas avaliadas foram pesadas, em uma balança digital da marca Welmy, para se obter um parâmetro para o equipamento de força muscular relativa. A calibração do equipamento foi feita por um peso de $5 \mathrm{Kgf}$, para que 0 equipamento pudesse ser ajustado, a reconhecer a força de preensão manual desenvolvida pelas idosas, antes da realização da avaliação.

Durante a avaliação da força de preensão manual, cada idosa foi posicionada sentada em uma cadeira, utilizando 0 membro superior dominante apoiado, com o antebraço em posição neutra, e cotovelo flexionado a $90^{\circ}$ ADM. Ao comando do avaliador, conforme o início do registro pelo computador, cada idosa realizou a contração muscular isométrica resistida, dos flexores dos dedos, com duração de 5 segundos. Foram realizadas três repetições com intervalo de um minuto. Foi considerada para a avaliação das idosas, a força muscular máxima de preensão manual em Kgf, das três repetições.

O programa cinesioterapêutico validado, ${ }^{9}$ foi realizado 2 vezes por semana, com duração de 40 minutos por dia, durante 2 meses, com as idosas estando vestindo roupas leves, e calçadas, em um ambiente arejado e amplo. Em cada um dos dois dias na semana, foi desenvolvido 10 minutos de alongamentos gerais MMSS, 20 minutos de exercícios ativos resistido, com 0 uso do equipamento digiflex, e bolas pequenas para mãos, para 0 fortalecimento dos flexores superficiais e profundos dos dedos, no movimento de flexão dos dedos. O fortalecimento dos músculos flexores radial e ulnar do carpo, e extensores radial longo e curto, e ulnar do carpo foram realizados com o uso de carga de $1 \mathrm{~kg}$, nos movimentos articulares de flexão e extensão do punho.

Foi também realizado o fortalecimento dos músculos bíceps e tríceps braquial, com cargas de 1 $\mathrm{Kg}$ e $2 \mathrm{Kg}$, nos movimentos de flexão e extensão de cotovelos. Para o fortalecimento dos músculos supra espinhoso, deltoide fibras médias, e peitoral maior, foi usado cargas de $2 \mathrm{Kg}$ e $3 \mathrm{Kg}$, para os movimentos articulares de abdução e adução do ombro; e as cargas de $2 \mathrm{Kg} \mathrm{e} 3 \mathrm{Kg}$ foram também utilizadas para o fortalecimento dos músculos deltoide anterior e posterior, nos movimentos articulares de flexão e extensão do ombro.

Para a finalização do programa de exercícios foram realizados 10 minutos de alongamentos gerais MMSS. Em relação às 10 idosas pertencentes à Associação Comunitária da Vila Cruzado, foi feita apenas a avaliação da força muscular máxima de preensão manual em um intervalo de 2 meses, entre a primeira e a última avaliação, tendo estas não sido submetidas ao programa cinesioterapêutico, pois fizeram parte do grupo controle (GC).

Este estudo foi baseado na Resolução 466/12 do Conselho Nacional de Saúde ${ }^{12}$ que regulamenta as pesquisas envolvendo seres humanos, respeitando-se a Convenção de Helsinki em 2008. ${ }^{13}$ O projeto de 
pesquisa foi aprovado pelo Comitê de Ética em Pesquisa em Humanos, da Universidade CEUMA (CEP), com o número 289.169/2013.

Os dados referentes à coleta de dados foram tabulados em uma planilha do programa Microsoft Office Excel 2007®, e transferidos para análise com 0 programa estatístico SPSS 18.0.

$\mathrm{Na}$ estatística descritiva, as variáveis quantitativas com distribuição simétrica são apresentadas em média e desvio padrão $e$ as assimétricas em mediana, mínimo e máximo. Para a análise do efeito do programa cinesioterapêutico na força de preensão manual de idosas e na comparação intergrupos foi utilizado o teste de Wilcoxon. Na análise da relação entre força de preensão manual e faixa etária das idosas submetidas ao programa cinesioterapêutico foi utilizado o Kruskal-Wallis, sendo considerado significativo $p<0,05$.

\section{Resultados}

Participaram do estudo 30 idosas, sendo 20 alocadas para o grupo submetido a um protocolo de cinesioterapêutico e 10 para o grupo que não realizou a intervenção. Ao serem comparados os grupos, não foi verificada diferença significativa com relação a nenhuma característica (tabela 1).

A mediana da força de preensão manual das idosas antes da aplicação do programa cinesioterapêutico foi $11,8(9,5-21,6)$ e após a intervenção foi 13,6 (12,420,1). O teste de Wilcoxon foi convertido em um escore $z$ de - 3,62 com uma probabilidade associada de 0,000. Assim, pode-se afirmar que o programa cinesioterapêutico melhora a força de preensão manual de idosas (figura 1).

A tabela 2 mostra a comparação da força muscular de preensão das idosas do grupo submetido ao protocolo cinesioterapêutico e do grupo que não sofreu nenhuma intervenção. Foi verificada diferença estatisticamente significativa apenas no grupo submetido ao programa cinesioterapêutico.

Para avaliar a relação entre força de preensão manual e faixa etária das idosas submetidas ao programa cinesioterapêutico, como a amostra era pequena e os dados não estavam distribuídos uniformemente, foi realizado 0 Kruskal-Wallis. A mediana da força muscular manual do grupo com faixa etária entre 61 e 70 anos foi 14,1 (12,7-15,5), entre 71 e 80 anos foi $16,2(13,7-18,7)$ e entre 81 e 90 foi 14,2 (12,7-20,1). Essa diferença apresentou valor de probabilidade associada de 0,69. Portanto, concluiu-se que não existem diferenças significativas na força muscular de idosas ativas por faixa etária.

\section{Discussão}

Apesar de neste estudo, o programa cinesioterapêutico realizado com as 30 idosas, ter sido desenvolvido apenas durante 02 meses, os resultados mostraram que houve um aumento da força de preensão manual, com 02 avaliações efetuadas, considerando-se a força máxima de 03 repetições realizadas em cada avaliação. Em contrapartida 0 
Tabela 1 - Características de idosas submetidas a protocolo cinesioterapêutico e de idosas sem intervenção. São Luís, 2013.

\begin{tabular}{|c|c|c|c|}
\hline Características & Intervenção (n=20) & Controle $(n=10)$ & $\mathbf{p}$ \\
\hline $\begin{array}{ll}\text { ldade, mediana (min- } \\
\text { máx) }\end{array}$ & $73(69-89)$ & $72(61-80)$ & $0,16^{*}$ \\
\hline Peso, média ( $\pm \mathrm{DP})$ & $57,6 \pm 11,3$ & $58,1 \pm 9,8$ & $0,45^{\star *}$ \\
\hline
\end{tabular}
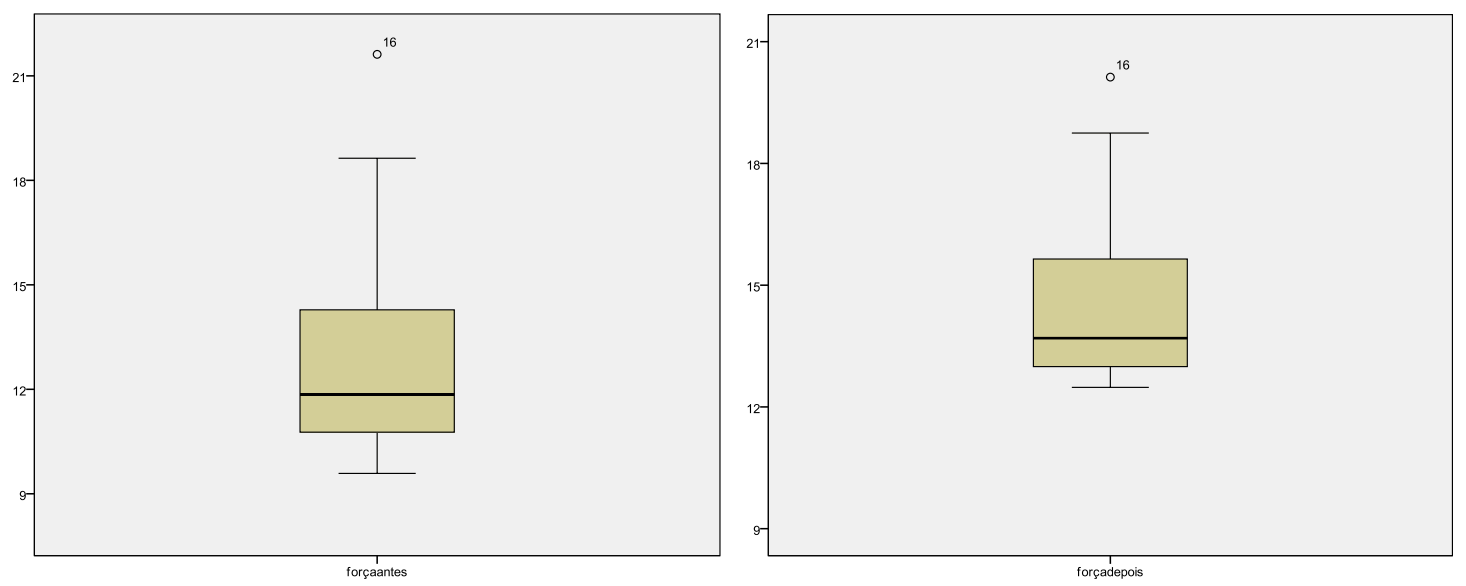

Figura 1 - Efeito de um programa cinesioterapêutico sobre a força de preensão manual de idosas. São Luís- MA, 2013.

Tabela 2 - Comparação da força muscular de preensão manual de idosas ativas e sedentárias. São Luís-MA, 2013

\begin{tabular}{lccccc}
\hline & Antes $^{*}$ & Min - máx & Depois $^{*}$ & Min-máx & $\mathbf{p}$ \\
\hline Ativas & 11 & $9,5-21,6$ & 13,6 & $12,4-20,1$ & 0,00 \\
Sedentárias & 11,7 & $5,1-20,2$ & 12,1 & $6,5-19,3$ & 0,38 \\
\hline
\end{tabular}


estudo feito por Déa et al. ${ }^{14}$, com 85 idosos com e sem depressão, com aplicação de um programa de ginástica, durante um ano, proporcionou um pequeno ganho de força de preensão manual, tendo sido feitas 04 avaliações durante 0 período, e considerada a força máxima.

O estudo desenvolvido com as 20 idosas no Clube de Repouso Irmã Dulce, para aumento da força de preensão manual, ocorreu 02 vezes por semana, com duração de 40 minutos por dia. Souto et al. ${ }^{15}$, ao comparar grupos de idosas ativas e sedentárias com relação à força muscular de membros superiores e inferiores mostrou que idosas ativas praticantes de exercícios físicos, 02 vezes por semana, com duração de 01 hora por dia, apresentaram maior força muscular que as idosas sedentárias.

Neste estudo as 30 idosas participantes, sendo 20 idosas que participaram do grupo experimental, e 10 idosas que participaram do grupo controle, apresentaram uma mediana de idade entre 72 e 73 anos, tendo o grupo experimental sido submetido a um programa cinesioterapêutico, durante 02 meses, que proporcionou um aumento da força de preensão manual. Estudo realizado por Rosa et al. ${ }^{16}$, com um grupo de 20 idosas, com média de idade de 67 anos, sendo 10 participantes de um grupo experimental, com prática de Pilates, 02 a 04 vezes por semana, durante um período de 02 meses a 02 anos, e 10 sedentárias; apresentou apenas para o grupo experimental um ganho de força muscular.

No estudo realizado não houve diferenças estatisticamente, na relação da idade das idosas com a prática do programa cinesioterapêutico, e sem a prática do programa cinesioterapêutico. Estudo realizado por Krug et al. ${ }^{17}$, mostrou que idosos com menor idade, desenvolvem uma atividade física com maior duração, do que idosos com idade mais avançada. Em concordância com Krug et al. ${ }^{17}$, outro estudo desta vez realizado por Livramento et al. ${ }^{18}$, mostrou que quanto maior for a idade de idosas, menor será a participação destas em atividade físicas.

Neste estudo, houve aumento da força muscular de preensão manual das idosas submetidas a um programa cinesioterapêutico, tendo trazido benefícios físicos a estas, enquanto que as idosas não submetidas ao programa cinesioterapêutico, não apresentaram aumento da força de preensão manual de forma significativa durante 0 mesmo período. Outro estudo com idosas de grupos de convivência realizado por Lopes et al. ${ }^{19}$, mostrou que programas que visem 0 desenvolvimento físico de idosas se faz importante, para que estas possam se manter ativas, mesmo com idade mais avançada. Corroborando com estes estudos, Azambuja et al. ${ }^{20}$ mostraram que programas de exercícios físicos devem ser planejados e executados para beneficiarem a saúde de idosas. Alencar et al. ${ }^{21}$, também mostraram em um estudo com 30 idosas, com idade entre 60 e 87 anos, que as idosas sedentárias apresentam menores níveis de avaliação funcional quanto à força muscular. 
Estudo realizado por Silva et al. ${ }^{7}$, com 50 idosos do sexo masculino e feminino, mostrou que as pessoas praticantes de atividade física regular, desenvolvem melhor nível de aptidão física, quando comparados a idosos sedentários. Neste estudo realizado com 30 pessoas idosas, somente participaram mulheres; as quais, apresentaram maior força muscular de preensão manual, as que foram submetidas ao programa cinesioterapêutico durante 02 meses. Outro estudo realizado por Araújo et al. ${ }^{21}$ mostrou que 0 treinamento resistido é capaz de melhorar a força muscular de idosos, concordando com este estudo realizado com idosas de grupo de convivência, vinculadas ao Clube de Repouso Irmã Dulce.

\section{Conclusão}

Concluiu-se neste estudo que idosas de grupos de convivência submetidas a um programa cinesioterapêutico, mesmo que durante um curto período, apresentam aumento da força de preensão manual.

A cinesioterapia do tipo ativo resistido mostrou ser uma importante ferramenta para o ganho de força muscular em idosas.

Entretanto sugere-se que outros estudos com maior duração e maior quantidade de idosas participantes, sejam realizados, para que possam ser obtidos outros resultados, que proporcionem mais referências sobre esta crescente parcela da população.

\section{Referências}

1. Meneses DLP, Silva FJG Jr, Melo HSF, Silva JC, Luz VLES, Figueiredo MLF. A dupla face da velhice: o olhar de idosos sobre 0 processo de envelhecimento. Enferm Foco. 2013;4(1):15-8.

2. Torres GV, Reis LA, Reis LA, Fernandes MH. Qualidade de vida e fatores associados em idosos dependentes em uma cidade do interior do Nordeste. J Bras Psiquiatr. 2009;58(1):39-44.

3. Moraes K, Correa CS, Pinto RS, Schuch F, Rodaelli R, Gaya A, et al. Efeitos de três programas de treinamento de força na qualidade de vida de idosas. Rev Bras Ativ Fis e Saúde. 2012;17(3):181-7.

4. Pedrinelli A, Garcez-Leme LE, Nobre RSA. O efeito da atividade física no aparelho locomotor do idoso. Rev Bras Ortop. 2009;44(2):96-101.

5. Albuquerque IS, Emmanouilidis A, Ortolan T, Cardoso DM, Gass R, Jost $R T$, et al. Capacidade funcional submáxima e força muscular respiratória entre idosas praticantes de hidroginástica e dança: um estudo comparativo. Rev Bras Geriatr Gerontol. 2013;16(2):327-36.

6. Dallasta VC, Radunz RL. Os pilates na reabilitação fisioterapêutica em idosos. Rev Uningá Review. 2013;15(1):44-7.

7. Silva MF, Goulart NBA, Lanfedini FJ, Marcon M, Dias CP. Relação entre os níveis de atividade física e qualidade de vida de idosos sedentários e fisicamente ativos. Rev Bras Geriatr Gerontol. 2012;15(4):635-42.

8. Alencar NA, Souza JV Jr, Aragão JCB, Ferreira MA, Dantas E. Nível de atividade física, autonomia funcional e qualidade de vida em idosas ativas e sedentárias. Fisioter Mov. 2010;23(3):473-81.

9. Montenegro SMRS, Silva CAB. Os efeitos de um programa de fisioterapia como promotor de saúde na capacidade funcional de mulheres idosas institucionalizadas. Rev Bras Geriatr Gerontol. 2007;10(2):161-78. 
10. Rebellato JR, Castro AP, Chan A. Quedas em idosas institucionalizados: características gerais, fatores determinantes e relações com a força de preensão manual. Acta Ortop Bras. 2007;15(3):151-4.

11. Thomas JR, Nelson JK, Silverman SJ. Métodos de pesquisa em atividade física. 5 ${ }^{\mathrm{a}}$ ed. Porto Alegre: Artmed; 2007.

12. Conselho Nacional de Saúde. Resolução no 466, de 12 de dezembro de 2012. Homologa a Resolução CNS no 466, de 12 de dezembro de 2012, nos termos do decreto de Delegação de Competência de 12 de novembro de 1991. Diário Oficial da União, Brasília (DF); 2013 jun 13; Seção 1:59.

13. World Medical Association. World Medical Association Declaration of Helsinki: ethical principles for medical research involving human subjects. 59th ed. Seoul: WMA General Assembly, 2008.

14. Déa VHSD, Duarte E, Rebelatto JR, Castro AP. Força muscular de idosos com e sem depressão participantes de um programa de ginástica. Acta Ortop Bras. 2009;17(6):322-5.

15. Souto PPC, Bandeira TF, Sandoval RA. Força muscular de membros superiores e inferiores: estudo correlacional comparativo entre grupos de idosas. Estudos. 2010;37(1/2):83100.

16. Rosa KB, Liposcki D, Waltrick $T$, Slongo A. Qualidade de vida e avaliação funcional em idosos praticantes de pilates e idosos sedentários. RIES. 2013;2(1):18-28.

17. Krug RR, Conceição JCR, Garcia GS, Streit IA, Mazo GZ. Idosos praticantes de atividades físicas: relação entre gênero e idade. Biomotriz. 2011;5(1):1-16.
18. Livramento GA, Fagundes $P$, Winter $G$, Bernardes V, Krause M. Estudo longitudinal do nível de atividade física de mulheres idosas. Rev Bras Ativ Fis e Saúde. 2012;17(6):552-61.

19. Lopes MA, Krug RR, Mazo GZ, Bonetti A. Percepção de idosas longevas sobre atitudes positivas diante da prática de atividade física: um estudo em grupo focal. Sau \& Transf Soc. 2013;4(3):91-7.

20. Azambuja CR, Machado RR, Santos DL. Correlação entre estilo de vida e nível de atividade física de idosas sedentárias e ativas. R Bras Ci e Mov. 2013;21(3):142-9.

21. Alencar NA, Souza JV Jr, Aragão JCB, Ferreira MA, Dantas E. Perfil do nível de atividade física e autonomia funcional de idosas. Fisioter Mov. 2011;8(1):21-8.

22. Araújo MLM, Fló CM, Muchale SM. Efeitos dos exercícios resistidos sobre - equilíbrio e a funcionalidade de idosos saudáveis: artigo de atualização. Fisioter Pesq. 2010;17(3):277-83. 